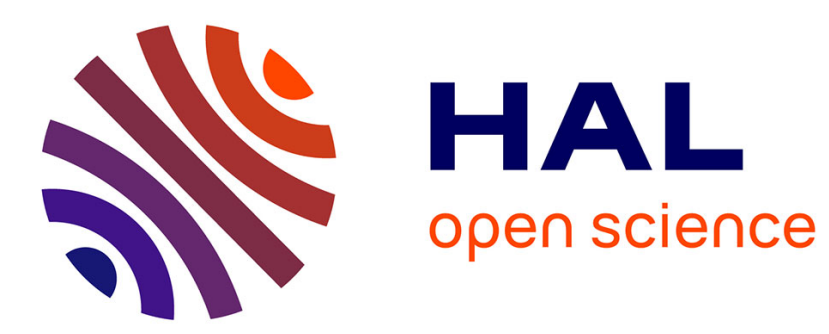

\title{
Distributed feedback control of a fractional diffusion process
}

\author{
Ahmed Maidi, Jean-Pierre Corriou
}

\section{To cite this version:}

Ahmed Maidi, Jean-Pierre Corriou. Distributed feedback control of a fractional diffusion process. International Journal of Dynamics and Control, 2019, 7, pp.1091-1100. 10.1007/s40435-018-0493-0 . hal-02130574

\section{HAL Id: hal-02130574 https://hal.science/hal-02130574}

Submitted on 27 Nov 2019

HAL is a multi-disciplinary open access archive for the deposit and dissemination of scientific research documents, whether they are published or not. The documents may come from teaching and research institutions in France or abroad, or from public or private research centers.
L'archive ouverte pluridisciplinaire HAL, est destinée au dépôt et à la diffusion de documents scientifiques de niveau recherche, publiés ou non, émanant des établissements d'enseignement et de recherche français ou étrangers, des laboratoires publics ou privés. 
archives-ouvertes

\title{
Distributed feedback control of a fractional diffusion process
}

\author{
Ahmed Maidi, Jean-Pierre Corriou
}

\section{To cite this version:}

Ahmed Maidi, Jean-Pierre Corriou. Distributed feedback control of a fractional diffusion process. International Journal of Dynamics and Control, Springer Berlin Heidelberg, In press, 10.1007/s40435018-0493-0 . hal-02130574

\section{HAL Id: hal-02130574 \\ https://hal.archives-ouvertes.fr/hal-02130574}

Submitted on 27 Nov 2019

HAL is a multi-disciplinary open access archive for the deposit and dissemination of scientific research documents, whether they are published or not. The documents may come from teaching and research institutions in France or abroad, or from public or private research centers.
L'archive ouverte pluridisciplinaire HAL, est destinée au dépôt et à la diffusion de documents scientifiques de niveau recherche, publiés ou non, émanant des établissements d'enseignement et de recherche français ou étrangers, des laboratoires publics ou privés. 


\title{
Distributed feedback control of a fractional diffusion process
}

\author{
Ahmed Maidi \\ Jean-Pierre Corriou
}

\begin{abstract}
In this paper, a control law that enforces an output tracking of a fractional diffusion process is developed. The dynamical behavior of the process is described by a space-fractional parabolic equation. The objective is to force a spatial weighted average output to track its specified output by manipulating a control variable assumed to be distributed in the spatial domain. The state feedback is designed in the framework of geometric control using the notion of the characteristic index. Then, under the assumption that the fractional diffusion process is a minimum phase system, it is shown that the developed control law guarantees exponential stability in $L_{2}$-norm for the resulting closed loop system. Numerical simulations are performed to show the tracking and disturbance rejection capabilities of the developed controller.
\end{abstract}

Keywords Distributed parameter system - fractional partial differential equation - fractional diffusion - late lumping - geometric control characteristic index.

\section{Introduction}

In the case of Fractional Differential Equations (FDEs), the order of the derivative is non-integer, allowing them more flexibility to accurately model dynamical systems than usual Ordinary or Partial Differential Equations (ODEs or PDEs) [1-6]. This success is mainly due to the remarkable nonlocal property of the fractional derivative $[2,7]$. Indeed, in some cases, the evolution of a physical variable depends both on its current and past values, so that this dynamical behavior can be captured using the fractional derivative $[2,8,9]$. Consequently, FDEs represent an excellent alternative modeling approach to integer differential equations (IDEs, i.e. ODES and PDEs) and with the considerable development of the fractional calculus [10], this kind of equation is nowadays extensively used in some engineering fields [9-11], particularly in system and control theories.

In particular, the model of nuclear reactors is based on the neuron transport equation. Classicaly, the neutron diffusion equation is modelled by an integer order PDE equation based on Fick's law. However, this predicts an infinite speed of propagation of neutrons which is non physical. The introduction of anomalous diffusion [12] as subdiffusion results in a fractional order model of neuron transport and allows to better represent the heterogeneous core of nuclear reactors [13,14].

Non-equilibrium processes designate a class of complex dynamical systems common in transport-reaction phenomena [2] where heat and mass transfer must be considered instead of simple equilibrium. Thus, anomalous convection, anomalous diffusion and anomalous convection-diffusion-reaction phenomena, to name a few, characterize the dynamical behavior of some complex biological, chemical and heat processes. The mathematical modeling of these anomalous dynamical processes leads to fractional partial differential equations (FPDEs) that are able to describe accurately their behavior [2-5]. Mainly, three types of FPDEs are to be distinguished: time-FPDEs, space-FPDEs and time-spaceFPDEs. Both mathematical and physical classifications of these FPDEs are reviewed in [15]. Dynamical processes described by FPDEs are classified as distributed parameter systems or infinite dimensional systems.

Compared to the control theory of FPDEs, the control theory of integer partial differential equations (IPDEs) has been has been developed for a long time [16-18] and two approaches have emerged: early and late lumping approaches [18-20]. The early lumping approach consists in reducing the infinite dimensional system (IPDEs) to a finite dimensional one, that is, a lumped parameter 
system (described by ordinary differential equations) using different model reduction techniques $[18,19,21]$. It is well known that the early lumping approach often masks the distributed nature of the original system and leads to high-dimensional controller with limited performance $[18,20,22,23]$, and its usage is recommended for parabolic systems [23]. The late lumping approach consists in using the infinite dimensional model (IPDEs), both in the analysis and controller design stages, without any reduction or approximation. This approach, which can be adopted for both hyperbolic and parabolic systems, allows to preserve the distributed nature of the infinite dimensional system and to enhance the closed loop performance. Excellent reviews of the different control techniques developed in the literature for IPDEs can be found in $[17,20,23]$.

Chen [24] has written a review about the control of various systems described by fractional order models in general with future perspectives and many open problems, however limited to the case of fractional ordinary differential equations. A remarkable example is the CRONE controller [25] and real life applications include car suspension control [26], flexible transmission, hydraulic actuator. The application of fractional order PID control to power systems is reported by [27].

However, the control design of FPDEs is still relatively undeveloped field except a few recent reported results in the area of optimal control [28-30]. Stabilization and output tracking problems remain unexplored fields. Hence, handling this two central control problems by extending the late lumping approach to FPDEs constitutes an interesting challenge for the control community. To our knowledge, the current work represents a first or initial attempt to tackle the control of fractional diffusion process following the late lumping approach. This SFPDE, classified mathematically as a space-fractional parabolic equation [15], is commonly used as an effective mathematical tool to characterize the evolution of many anomalous dynamical processes [2,31-34].

The geometric control technique presents the advantage that the FPDE model can be easily used in control design without any approximation $[22,35,36]$. Consequently, the distributed nature of the process is preserved, yielding a controller with improved performance. Thus, in this manuscript, the geometric control technique is used to design a distributed feedback control for the fractional diffusion process. The Caputo version $[9,37]$ is selected in this work for the definition of the spatial fractional derivative that describes the anomalous diffusion phenomenon. Based on the reasonable assumption that the functions that characterize both the actuation and the sensing distributions are not orthogonal, a control law of distributed nature is derived based on the concept of the characteristic index [22]. Then, by exploiting the minimum phase property of the diffusion process, it is demonstrated that the developed control law yields an exponentially stable closed loop system in the $L_{2}$-norm. The output tracking and disturbance rejection performance of the developed control is evaluated through numerical simulation, and both punctual and spatial weighted average outputs are considered. The closed loop system is simulated using the method of lines and the Caputo's fractional derivative is approximated with splines $[38,39]$.

The content of the paper is structured as follows: In Section 2, the control problem of the fraction diffusion process is formulated. Section 3 is devoted to the design of the distributed control law, in the framework of geometric control theory, and to the closed loop stability issue. An application example with numerical simulation results is reported in Section 4. Finally, Section 5 concludes the paper.

\section{Control problem}

Let us consider the fractional diffusion process described by the following one-dimensional space-fractional parabolic equation

$\frac{\partial x(z, t)}{\partial t}={ }_{0} D_{z}^{\alpha} x(z, t)+b(z) u(t), \quad 0<z<1$

with the following Dirichlet boundary conditions

$x(0, t)=x_{0}(t)$

$x(1, t)=x_{1}(t)$

and the initial condition

$x(z, t)=x^{*}(z)$

In this fractional diffusion model, $z \in[0,1]$ and $t \in[0,+\infty[$ represent the space and time variables, respectively. $x(z, t) \in L^{2}([0,1])$ is the state and $u(t) \in$ $L^{2}([0,+\infty[; \Re)$ is the manipulated variable assumed to be distributed along the spatial domain $[0,1] . b(z) \in$ $L^{2}([0,1])$ is a smooth function that characterizes the distribution of the input $u(t) . x_{0}(t)$ and $x_{1}(t)$ are the state values at the boundaries $z=0$ and $z=1$, respectively, and $x^{*}(z) \in L^{2}([0,1])$ is a given initial profile. ${ }_{0} D_{z}^{\alpha}$ is the bounded spatial fractional derivative operator where $\alpha$ is the order of the fractional derivative. $L^{2}($. ) and $\Re$ are the space of the square-integrable functions on the interval $[0,1]$ and the set of real numbers, respectively.

Remark 1 This paper deals with fractional diffusion process, that is, $\alpha \in] 1,2[$ [15]. 
Remark $2 L^{2}([0,1])$ is the space of square-integrable functions [40], defined on the domain [0,1], endowed with the inner product

$\langle f(z), g(z)\rangle=\int_{0}^{1} f(z) g(z) d z, \forall f(z), g(z) \in L^{2}([0,1])$

and the norm

$\|f(z)\|=\sqrt{\langle f(z), f(z)\rangle}$

The objective consists in designing a control law $u(t)$ so that the following output

$y(t)=\int_{0}^{1} c(z) x(z, t) d z$

can track a desired reference $y^{d}(t)$. In equation (7), the smooth function $c(z) \in L^{2}([0,1])$ characterizes the location of the measurements. The functions $b(z)$ and $c(z)$ are chosen so that both desired performance and control design specifications are met. For the formulated control problem, the following assumption is made.

Assumption 1 The smooth functions $b(z)$ and $c(z)$ are not orthogonal [40], that is,

$\langle b(z), c(z)\rangle=\int_{0}^{1} c(z) b(z) d z \neq 0$

Remark 3 The fractional diffusion process (1)-(4) is a minimum-phase system. This property can be checked by simulation [22]. The minimum-phase property implies that the zero dynamics is stable.

For the formulated control problem, a definition of the fractional derivative must be specified. The different definitions proposed in the literature are reviewed by [37]. It is worth noting that the Grnwald-Letnikov, Riemann-Liouville and Caputo derivatives remain the widely used definitions $[9,10,37]$. Nevertheless, the Caputo derivative presents some advantages [41]: $(i)$ the usual properties of integer calculus are preserved, for instance the Caputo derivative of a constant is zero, (ii) the definition of the Caputo derivative involves an integral of an integer derivative, thus an accurate numerical approximation of this term can be achieved using powerful algorithms [38]. In this work, the Caputo derivative definition is used.

Definition 1 The spatial Caputo derivative of order $\alpha$ for $x(z, t)$ with the lower limit zero is defined as follows

$$
\begin{aligned}
{ }_{0} D_{z}^{\alpha} x(z, t) & =\frac{\partial^{\alpha} x(z, t)}{\partial z^{\alpha}} \\
& =\frac{1}{\Gamma(n-\alpha)} \int_{0}^{z} \frac{\partial^{n} x(z, t)}{\partial z^{n}}(z-\xi)^{n-\alpha-1} d \xi
\end{aligned}
$$

Note that, in equation (10), $n$ is the smallest integer greater than $\alpha$, that is, $n-1<\alpha \leq n$, and $\Gamma($.$) is the$ gamma function [9].

Remark 4 For the fractional diffusion process (1), as the order $\alpha \in] 1,2[$, hence $n=2$, and according Definition 1, the Caputo derivative is defined as

${ }_{0} D_{z}^{\alpha} x(z, t)=\frac{1}{\Gamma(2-\alpha)} \int_{0}^{z} \frac{\partial^{2} x(z, t)}{\partial z^{2}}(z-\xi)^{1-\alpha} d \xi$

\section{Geometric control of fractional diffusion process}

The fractional diffusion equation (1) possesses a particular propriety related to the order of the fractional derivative $\alpha$. Salehi and Schiesser [41] investigated through simulation runs the nature of the solution of this equation with respect to $\alpha$. It is shown that by changing the order $\alpha$ from 1 to 2 , equation (1) changes from hyperbolic (convective) to parabolic (diffusion) equation, thus for intermediate values, that is, for $\alpha \in] 1,2[$, the equation is hyperbolic-parabolic (convective-diffusive). This propriety motivates, as pointed out in the introduction, to tackle the formulated control problem according to the late lumping approach. Among the control design techniques based on the direct use of the PDE model, geometric control presents some remarkable advantages [42]. This control technique is adopted here to design the control law $u(t)$ that achieves the output tracking.

The design of a geometric control law is based on the characteristic index introduced by [22], which represents a generalization of the well-known notion of the relative degree [43] of a finite dimensional system to an infinite dimensional one.

\subsection{Distributed feedback control design}

In this subsection, the distributed control law $u(t)$ that ensures output tracking is derived based on the concept of the characteristic index. Thus, the first time derivative of the controlled output (7) reads as

$$
\begin{aligned}
\frac{d y(t)}{d t} & =\int_{0}^{1} c(z) \frac{\partial x(z, t)}{\partial t} d z \\
& =\int_{0}^{1} c(z)\left({ }_{0} D_{z}^{\alpha} x(z, t)+b(z) u(t)\right) d z \\
& =\int_{0}^{1} c(z){ }_{0} D_{z}^{\alpha} x(z, t) d z+\left[\int_{0}^{1} c(z) b(z) d z\right] u(t)
\end{aligned}
$$


Hence, from equation (14), it can be seen that $u(t)$ appears linearly in the first time derivative of $y(t)$. Hence, taking into account Assumption 1, it follows that the characteristic index $\sigma=1$. This means that a control $u(t)$ that preserves the characteristic index in closed loop can be designed. Consequently, a first-order dynamical behavior can be achieved between the desired reference $y^{d}(t)$ and the controlled output $y(t)$, that is,

$\tau \frac{d y(t)}{d t}+y(t)=y^{d}(t)$

where $\tau$ is the desired time constant in closed loop. In the subsequent development, it is assumed that the derivative of the desired reference $y^{d}(t)$ satisfy the following condition

$\left|\frac{d y^{d}(t)}{d t}\right| \leq M e^{-\omega t}, \quad M \geq 1, \omega>0$

where $|$.$| is the standard Euclidean norm.$

Then, substituting (14) into (15) and solving for $u(t)$ the resulting equation, the following feedback law follows

$u(t)=\frac{y^{d}(t)-y(t)+\tau \int_{0}^{1} c(z){ }_{0} D_{z}^{\alpha} x(z, t) d z}{\tau \int_{0}^{1} c(z) b(z) d z}$

which can be equivalently expressed as

$u(t)=\frac{y^{d}(t)-y(t)+\tau \int_{0}^{1} c(z) \frac{\partial^{\alpha} x(z, t)}{\partial z^{\alpha}} d z}{\tau \int_{0}^{1} c(z) b(z) d z}$

The stability of the resulting closed loop is investigated in the following subsection.

Remark 5 In the present work, the state is assumed to be perfectly known. In real practice, the derivative approximation in the control law (18) is significantly influenced by measurement noise. This problem is addressed in Section 3.3.

\subsection{Closed loop stability issue}

From (15), it is concluded that the closed loop system is externally stable. In this section, we investigate the stability of the internal dynamics of the closed loop system, that is, the unobservable part of the dynamics from the output (7).
Thus, substituting (18) into (1) yields closed loop system which can be written under the abstract form

$$
\begin{aligned}
\frac{\partial x(z, t)}{\partial t} & =\mathcal{A} x(z, t)+\mathcal{B}\left(y^{d}(t)-y(t)\right) \\
x(0, t) & =x_{0}(t) \\
x(l, t) & =x_{l}(t) \\
x(z, 0) & =x^{*}(z)
\end{aligned}
$$

with

$$
\begin{aligned}
\mathcal{A} x(z, t) & ={ }_{0} D_{z}^{\alpha} x(z, t)+\frac{b(z) \int_{0}^{1} c(z) \frac{\partial^{\alpha} x(z, t)}{\partial z^{\alpha}} d z}{\int_{0}^{1} c(z) b(z) d z} \\
\mathcal{B} & =\frac{b(z)}{\tau \int_{0}^{1} c(z) b(z) d z}
\end{aligned}
$$

Note that the closed loop system (19)-(22) can be rewritten as the the interconnection of two subsystems

$$
\begin{aligned}
\frac{d y(t)}{d t} & =-\frac{1}{\tau} y(t)+\frac{1}{\tau} y^{d}(t) \\
\frac{\partial x(z, t)}{\partial t} & =\mathcal{A} x(z, t)+\mathcal{B} e(t) \\
x(0, t) & =x_{0}(t) \\
x(l, t) & =x_{l}(t) \\
x(z, 0) & =x^{*}(z)
\end{aligned}
$$

with

$e(t)=y^{d}(t)-y(t)$

Equations (25) and (26) represent the external and the internal dynamics, respectively.

By forcing the controlled output (7) to be zero, we obtain the following zero dynamics

$$
\begin{aligned}
\frac{\partial x(z, t)}{\partial t} & =\mathcal{A} x(z, t) \\
x(0, t) & =x_{0}(t) \\
x(l, t) & =x_{l}(t) \\
x(z, 0) & =x^{*}(z) \\
y(t) & =0
\end{aligned}
$$

Now, Remark 3 implies that the zero dynamics (31)(34) is stable. Consequently, the operator $\mathcal{A}$ generates a stable $C_{0}$-semigroup $U(t)$ [44], that is,

$\|U(t)\| \leq M_{x} e^{-\omega_{x} t}, \quad M_{x} \geq 1, \omega_{x}>0$ 
Remark 6 The zeroing of the output allows to conclude that the operator $\mathcal{A}$ generates a stable semigroup from the minimum-phase property of the diffusion system [22].

Thereafter, from equations (15) and (16), it follows that $e(t) \in L^{1}\left(\left[0,+\infty[, \Re)\left(L^{1}([0,+\infty[, \Re)\right.\right.\right.$ is the space of all integrable functions on the interval $[0,+\infty[)$, and since the time constant $\tau$ is positive, the tracking error is exponentially stable, that is,

$$
|e(t)| \leq M_{e} e^{-\omega_{e} t}, \quad M_{e} \geq 1, \omega_{e}>0
$$

where $|$.$| is the Euclidean norm.$

In this case, the mild solution of internal dynamics (26) is given by [45]

$x(z, t)=U(t) x(z, 0)+\int_{0}^{t} U(t-\xi) \mathcal{B} e(\xi) d \xi$

and therefore

$$
\begin{aligned}
\|x(z, t)\| & =\left\|U(t) x(z, 0)+\int_{0}^{t} U(t-\xi) \mathcal{B} e(\xi) d \xi\right\| \\
& \leq\|x(z, 0) U(t)\|+\left\|\int_{0}^{t} U(t-\xi) \mathcal{B} e(\xi) d \xi\right\| \\
& \leq\|x(z, 0)\| M_{x} e^{-\omega_{x} t} \\
& +\int_{0}^{t} M_{x} e^{-\omega_{x}(t-\xi)}\|\mathcal{B}\| M_{e} e^{-\omega_{e} \xi} d \xi \\
& \leq\|x(z, 0)\| M_{x} e^{-\omega_{x} t} \\
& +M_{x} M_{e}\|\mathcal{B}\| e^{-\omega_{x} t} \int_{0}^{t} e^{\left(\omega_{x}-\omega_{e}\right) \xi} d \xi
\end{aligned}
$$

Hence, if $\omega_{x}=\omega_{e}$, then

$$
\|x(z, t)\| \leq\left(\|x(z, 0)\| M_{x}+M_{x} M_{e}\|\mathcal{B}\| t\right) e^{-\omega_{x} t}
$$

and if $\omega_{x} \neq \omega_{e}$, we have

$$
\begin{aligned}
\|x(z, t)\| & \leq\|x(z, 0)\| M_{x} e^{-\omega_{x} t} \\
& +\frac{M_{x} M_{e}\|\mathcal{B}\|}{\omega_{x}-\omega_{e}}\left(e^{-\omega_{e} t}-e^{-\omega_{x} t}\right) \\
& \leq\left(\|x(z, 0)\| M_{x}+\frac{M_{x} M_{e}\|\mathcal{B}\|}{\left|\omega_{x}-\omega_{e}\right|}\right) e^{-\omega t}
\end{aligned}
$$

where $\omega=\min \left\{\omega_{x}, \omega_{e}\right\}$.

From (43) and (45), it follows that the internal dynamics of the closed loop system (19)-(22) is exponentially stable in the $L^{2}$ norm. Consequently the closed loop system is exponentially stable.

\subsection{Robustness}

The purpose of the present paper is to present a control law for a fractional diffusion process modelled by the FPDE (1). Thus, it was assumed in the design of the control law that the model is perfect and that the states are known. Obviously, this is not the case in actual practice and we will shortly explain how the control law can still be applied at the expense of additional features.

A first issue is that the model is never perfectly known and parameters are more or less uncertain. In the same way as in nonlinear geometric control [22,23, $42,43]$, an external input $v(t)$ can be added to the manipulated input to cope with these uncertainties as

$$
\begin{aligned}
& \begin{array}{c}
v(t)+y^{d}(t)-y(t)+\tau \quad \int_{0}^{1} c(z) \frac{\partial^{\alpha} x(z, t)}{\partial z^{\alpha}} d z \\
\hline
\end{array} \\
& \tau \int_{0}^{1} c(z) b(z) d z
\end{aligned}
$$

The external input $v(t)$ is defined by the following linear controller

$v(t)=\int_{0} \varepsilon(t-s)\left[y^{d}(s)-y(s)\right] d s$

where the kernel $c(t)$ is chosen in order to reach the desired performance, in particular using integral action to ensure regulation and tracking.

The second issue is that the states are not known. In that case, it is necessary to estimate the states by means of a state observer using some measurements. However, it was not done here as the observer design for a PDE [46-51] and even more for a FPDE is com-plex and would have deserved to be the subject of a totally different study. If measurements are noisy, the measurements should be first cleaned from the highfrequency noise by a low-pass filter, then an observer based on the FPDE should be used. In this manner, the main problems dealing with the robustness of the designed control law can be handled.

\section{Simulation results}

In this section, simulation runs are performed to evaluate the tracking and disturbance rejection capabilities of the designed infinite dimensional control law (46). The closed loop system is simulated using the method of lines [52] by assuming a spatial grid of $N=21$ points with a uniform spacing $\Delta z$ of the spatial interval $[0,1]$. The integral involved in the definition of the fractional 
spatial derivative (11), with $\alpha \in] 0,2[$, is approximated with splines as follows [39]:

$$
\begin{aligned}
\left.{ }_{0} D_{z}^{\alpha} x(z, t)\right|_{z=z_{j}} & =\left.\frac{\partial^{\alpha} x(z, t)}{\partial z^{\alpha}}\right|_{z=z_{j}} \\
& =\left.\frac{\Delta z^{2-\alpha}}{\Gamma(4-\alpha)} \sum_{k=0}^{j} \frac{\partial^{2} x(z, t)}{\partial z^{2}}\right|_{z=z_{k}} a_{j, k}
\end{aligned}
$$

where $z_{j}=j \Delta z(j=1 \ldots, N-1), a_{j, 0}=(j-1)^{3-\alpha}-$ $j^{2-\alpha}(j-3+\alpha), a_{j, j}=1$ and $a_{j, k}=(j-k+1)^{3-\alpha}-$ $2(j-k)^{3-\alpha}+(j-k-1)^{3-\alpha}$ for $k=1, \ldots, J-1$.

The second integer derivative involved in (49) is approximated using the following finite difference schemes [38]:

$\frac{\partial^{2} x\left(z_{0}, t\right)}{\partial z^{2}}=\frac{2 x\left(z_{0}, t\right)-5 x\left(z_{1}, t\right)+4 x\left(z_{2}, t\right)-x\left(z_{3}, t\right)}{\Delta z^{2}}$

and

$\frac{\partial^{2} x\left(z_{j}, t\right)}{\partial z^{2}}=\frac{x\left(z_{j+1}, t\right)-2 x\left(z_{j}, t\right)+x\left(z_{j-1}, t\right)}{\Delta z^{2}}$

for $j=1, \ldots, N-1$. The integral term involved in the control law is evaluated using the trapezoidal method.

To obtain a smooth control move when the desired reference $y^{d}(t)$ exhibits sudden fluctuations, it is proposed to use a filter to generate a reference trajectory with a specified dynamics. In this work, a first-order filter is used to generate the filtered reference $y_{f}^{d}(t)$, that is,

$\tau_{f} \frac{d y_{f}^{d}(t)}{d t}+y_{f}^{d}(t)=y^{d}(t)$

where $\tau_{f}$ is the filter time constant. In this case, the control law (18) takes the following form

$u(t)=\frac{y_{f}^{d}(t)-y(t)+\tau \int_{0}^{1} c(z) \frac{\partial^{\alpha} x(z, t)}{\partial z^{\alpha}} d z}{\tau \int_{0}^{1} c(z) b(z) d z}$

The simulation runs are carried out with $x^{*}(z)=0$, $\tau=0.25, \tau_{f}=0.1$ and the actuator distribution function $b(z)=z(1-z)$.

\subsection{Tracking problem}

The tracking problem is considered both for punctual and spatial weighted average outputs by assuming the following desired reference

$y^{d}(t)= \begin{cases}0 & t \leq 0.25 \\ 1 & t \leq 3 \\ 2 & t>3\end{cases}$

and boundary conditions

$x_{0}(t)=x_{l}(t)=0$

\section{- Punctual output case}

The first test concerns the case of a punctual output at location $z_{i}$. Thus, the function $c(z)$ is identified as a Dirac delta function, that is,

$c(z)=\delta\left(z-z_{i}\right), \quad 0<z_{i}<1$

hence the output to be controlled is defined as follows

$y(t)=x\left(z_{i}, t\right)$

In the simulation run, the position of the controlled output and the order of the fractional derivative are set to $z_{i}=0.75$ and $\alpha=1.3$, respectively.

- Spatial weighted average output case

In the second test, a spatial weighted average output with $c(z)=z$ is considered, i.e.,

$y(t)=\int_{0}^{1} z x(z, t) d z$

and the order of the fractional derivative is set to $\alpha=1.8$.

Figures 1 and 2 give the simulation results obtained for the punctual and the spatial weighted average cases, respectively. Figures 1a and 2a clearly show that the output tracking is achieved with smooth moves of the control (Figs. 1b and 2b). These results demonstrate the tracking capability of the developed control law (18). The evolutions (3D profile) of the state $x(z, t)$ are shown in Figures 1c and 2c for the punctual and the spatial weighted average cases, respectively.

\subsection{Disturbance rejection}

The disturbance rejection capabilities of the control law (18) are evaluated for smooth variations of the boundary conditions (2) and (3) assumed as disturbances. The simulation run consists in applying a set point followed by a smooth disturbance. The outputs and fractional orders assumed are the same as those in tracking problem. 
- Punctual output case

For the punctual output case, the smooth variation is applied at the left-hand boundary condition (2). The disturbance is defined as follows

$x_{0}(t)= \begin{cases}0 & t \leq 0.25 \\ 0.5\left(1-e^{-(t-3)}\right) & t \geq 3\end{cases}$

and the desired set point $y^{d}(t)=1$.

- Spatial weighted average output case

In this case, the disturbance applied at the righthand boundary condition (2) is defined as follows

$$
x_{1}(t)= \begin{cases}0 & t \leq 0.25 \\ 2\left(1-e^{-(t-3)}\right) & t \geq 3\end{cases}
$$

and $y^{d}(t)=2$.

The simulation results, given by Figures 3 and 4, demonstrate the capability of the control law (18) to reject the disturbance. Indeed, Figures $3 \mathrm{~b}$ and $4 \mathrm{~b}$ show that the control law (18) reacts to attenuate the disturbance observed at the boundary (Figures 3c and 4c) and its effect become invisible on the controlled output (Figures $3 \mathrm{a}$ and $4 \mathrm{a}$ ).

\section{Conclusion}

In this paper, the late lumping approach is extended to space-fractional distributed parameter systems. It shown that, for minimum phase systems, geometric control represents an interesting design approach of controllers of distributed nature. The case of the spacefractional parabolic equation that describes the anomalous diffusion is considered. Thus, by assuming a spatial weighted average output, a distributed control law that enforces the output tracking in closed loop is designed using the notion of the characteristic index. It is demonstrated, in the framework of semigroup theory, that the resulting closed loop system is exponentially stable in the $L_{2}$ norm. Both output tracking and disturbance rejection performance of the developed controller are successfully evaluated by performing simulation runs.

To the best knowledge of the authors, this attempt to tackle the control problem of the fractional distributed parameter systems, following the late lumping approach, represents the first contribution to this field. This motivates many possible directions of research to investigate this interesting and unexplored area.

\section{References}

1. Magin RL (2010) Fractional calculus models of complex dynamics in biological tissues. Computers and Mathematics with Applications 59(5):1586-1593
2. Klages R, Radons G, Sokolov IM (2008) Anomalous Transport. Foundations and Applications. Wiley-VCH Verlag GmbH \& Co. KGaA, Weinheim

3. Sierociuk D, Skovranek T, Macias M, Podlubny I, Petras I, Dzielinski A, Ziubinsk P (2015) Diffusion process modeling by using fractional-order models. Applied Mathematics and Computation 257(4):2-11

4. Chen W, Sun H, Zhang X, Korošak D (2010) Anomalous diffusion modeling by fractal and fractional derivatives. Computers and Mathematics with Applications 59(5):1754-1758

5. Žecová M, Terpák J (2015) Heat conduction modeling by using fractional-order derivatives. Applied Mathematics and Computation 257(4):365-373

6. Toledo-Hernandez R, Rico-Ramirez V, Iglesias-Silva GA, Diwekar UM (2014) A fractional calculus approach to the dynamic optimization of biological reactive systems. Part I: Fractional models for biological reactions. Chemical Engineering Science 117(9):217-228

7. Gómez Aguilar JF, Córdova-Fraga T, Tórres-Jiménez J, Escobar-Jiménez RF, Olivares-Peregrino VH, GuerreroRamírez GV (2016) Nonlocal transport processes and the fractional Cattaneo-Vernotte equation. Mathematical Problems in Engineering 2016, Article ID 7845874, 15 pages

8. Podlubny I (1994) Fractional Order Systems and Fractional Order Controllers. Report UEF-03-94, University of Technology, Kosice, Slovakia

9. Podlubny I, Thimann KV (1999) Fractional Differential Equations: An Introduction to Fractional Derivatives, Fractional Differential Equations, to Methods of Their Solution and Some of Their Applications. Academic Press, San Diego

10. Kilbas AA, Srivastava HM, Trujillo JJ (2006) Theory and Application of Fractional Differential Equations. North Holland Mathematics Studies, Elsevier, Amsterdam

11. Ionescu C, Lopes A, Copot D, Machado JAT, Bates JHT (2017) The role of fractional calculus in modeling biological phenomena: A review. Communications in Nonlinear Science and Numerical Simulation 51(10):141-159

12. Vyawahare VA, Nataraj PSV (2013) Fractional-order modeling of neutron transport in a nuclear reactor. Applied Mathematical Modelling 37(23):9747-9767

13. Vyawahare VA, Nataraj PSV (2018) Fractional-order Modeling of Nuclear Reactor: From Subdiffusive Neutron Transport to Control-oriented Models - A Systematic Approach. Springer Nature Singapore

14. Patra A, Saha Ray S (2017) On the Solution of the Nonlinear Fractional Neutron Point-Kinetics Equation with Newtonian Temperature Feedback Reactivity. Nuclear Technology 189(1):103-109

15. Li C, Chen A (2018) Numerical methods for fractional partial differential equations. International Journal of Computer Mathematics 95(6-7):1048-1099

16. Tzafestas SG, Stavroulakis P (1983) Recent advances in the study of distributed parameter systems. Journal of the Franklin Institute 315(5-6):285-305

17. Padhi R, Faruque Ali S (2009) An account of chronological developments in control of distributed parameter systems. Annual Reviews in Control 33(1):59-68

18. Christofides PD (2001a) Control of nonlinear distributed process systems: Recent developments and challenges. AIChE Journal 47(3):514-518

19. Ray WH (1989) Advanced Process Control. Butterworths, Boston

20. Meurer T (2013) Control of Higher-Dimensional PDEs. Flatness and Backstepping Designs. Springer-Verlag, Berlin 
21. Li HX, Qi C (2010) Modeling of distributed parameter systems for applications - A synthesized review from time-space separation. Journal of Process Control 20(8):891-901

22. Christofides PD, Daoutidis P (1996) Feedback control of hyperbolic PDE systems. AIChE Journal 42(11):30633086

23. Christofides PD (2001b) Nonlinear and Robust Control of PDE Systems: Methods and Applications to TransportReaction Processes. Birkhäuser, Boston

24. Chen YQ, Petras I, Xue D (1999) Fractional Order Control - A Tutorial. 2009 American Control Conference, St. Louis, USA

25. Oustaloup A, Levron F, Mathieu B, Nanot FM (2000) Frequency- band complex noninteger differentiator: characterization and synthesis, IEEE Trans. on Circuit and Systems - I: Fundamental Theory and Application, 47(1) 25-39

26. Oustaloup A, Moreau X, Nouillant M (1996) The CRONE suspension. Control Engineering Practice, 4(8):1101-1108

27. Nangrani SP, Bhat SS (2018) Fractional Order Controller for Controlling Power System Dynamic Behavior. Asian Journal of Control 20(1): 403-414

28. Jumari G (2007) Fractional Hamilton-Jacobi equation for the optimal control of nonrandom fractional dynamics with fractional cost function. Journal of Applied Mathematics and Computing 23(1-2):215-228

29. Wang J, Zhou Y (2011) Analysis of nonlinear fractional control systems in Banach spaces. Nonlinear Analysis 74(17):5929-5942

30. Wang J, Zhou Y, Wei W (2012) Optimal feedback control for semilinear fractional evolution equations in Banach spaces. Systems \& Control Letters 61(4):472-476

31. Gong C, Bao W, Tang G, Jiang Y, Liu J (2015) Computational challenge of fractional differential equations and the potential solutions: A survey. Mathematical Problems in Engineering 2015, Article ID 258265, 13 pages

32. Yang XJ, Machado JAT (2017) A new fractional operator of variable order: Application in the description of anomalous diffusion. Physica A: Statistical Mechanics and its Applications, 481(9):276-283

33. Copot D, Muresan C, De Keyser R, Ionescu C (2016) Fractional order modeling of diffusion processes: A new approach for glucose concentration estimation. In 2016 IEEE International Conference on Automation, Quality and Testing, Robotics (AQTR), Cluj-Napoca, Romania, May $19-21$

34. Copot D, Magin RL, De Keyser R, Ionescu C (2017) Data-driven modelling of drug tissue trapping using anomalous kinetics. Chaos, Solitons \& Fractals, 102(9):441-446

35. Maidi A, Corriou JP (2014b) Distributed control of nonlinear diffusion systems by input-output linearization. International Journal of Robust and Nonlinear Control 24(3):389-405

36. Maidi A, Corriou JP (2014a) Boundary geometric control of a linear Stefan problem. Journal of Process Control 24(6):939-946

37. de Oliveira EC, Machado JAT (2014) A review of definitions for fractional derivatives and integral. Mathematical Problems in Engineering 2014, Article ID 238459, 6 pages

38. Corriou JP (2010) Méthodes Numériques et Optimisation - Théorie et Pratique pour l'Ingénieur. Lavoisier, Tec. \& Doc., Paris
39. Sousa E (2011) Numerical approximations for fractional diffusion equations via splines. Computers and Mathematics with Applications 62(3):938-944

40. Atkinson K, Han W (2009) Theoretical Numerical Analysis. A Functional Analysis Framework. Springer-Verlag, New York

41. Salehi Y, Schiesser WE (2018) Numerical Integration of Space Fractional Partial Differential Equations. Vol 1 Introduction to Algorithms and Computer Coding in R. Morgan \& Claypool, California

42. Maidi A, Corriou JP (2016) Boundary geometric control of a nonlinear diffusion system with time-dependent spatial domain. Asian Journal Control 18(4):1259-1268

43. Isidori A (1995) Nonlinear Control Systems. SpringerVerlag, New York, Second edition

44. Pazy A (1983) Semigroup of Linear Operators and Applications to Partial Differential Equations. SpringerVerlag, New York

45. Curtain RF, Zwart H (1995) An Introduction to InfiniteDimensional Linear Systems Theory. Springer, New York

46. Castillo F, Witrant E, Prieur C, Dugard L (2013) Boundary observers for linear and quasi-linear hyper-bolic systems with application to flow control. Automat-ica 49(11):3180-3188

47. Gahlawat A, Peet M.M (2011) Designing observer-based controllers for PDE systems: A heat-conducting rod with point observation and boundary control. 50th IEEE Conference on Decision and Control CDC, Orlando, USA

48. Hasan A, Aamo OM, Krstic M (2016) Boundary observer design for hyperbolic PDE-ODE cascade systems. Automatica $68: 75-86$

49. Smyshlyaev A, Krstic M (2005) Backstepping observers for a class of parabolic PDEs. Systems \& Control Letters, 54(7):613-625

50. Wu HN, Wang JW (2013) Observer design and output feedback stabilization for nonlinear multivariable systems with diffusion PDE-governed sensor dynamics. Nonlinear Dyn. 72:615-628

51. Zobiri F, Witrant E, Bonne F (2017) PDE observer design for counter-current heat flows in a heat-exchanger. IFAC-PapersOnLine 50(1):7127-7132

52. Wouwer AV, Schiesser WE (2004) Simulation of distributed parameter systems using a Matlab-based method of lines toolbox: Chemical engineering applications. Industrial and Engineering Chemistry Research 43(14):3469-3477 


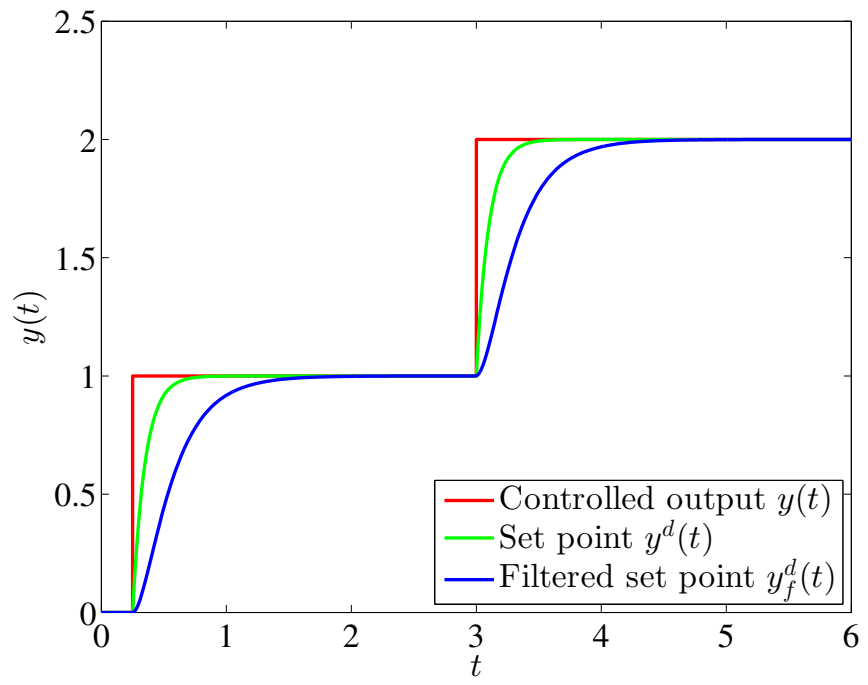

(a) Controlled output $y(t)$

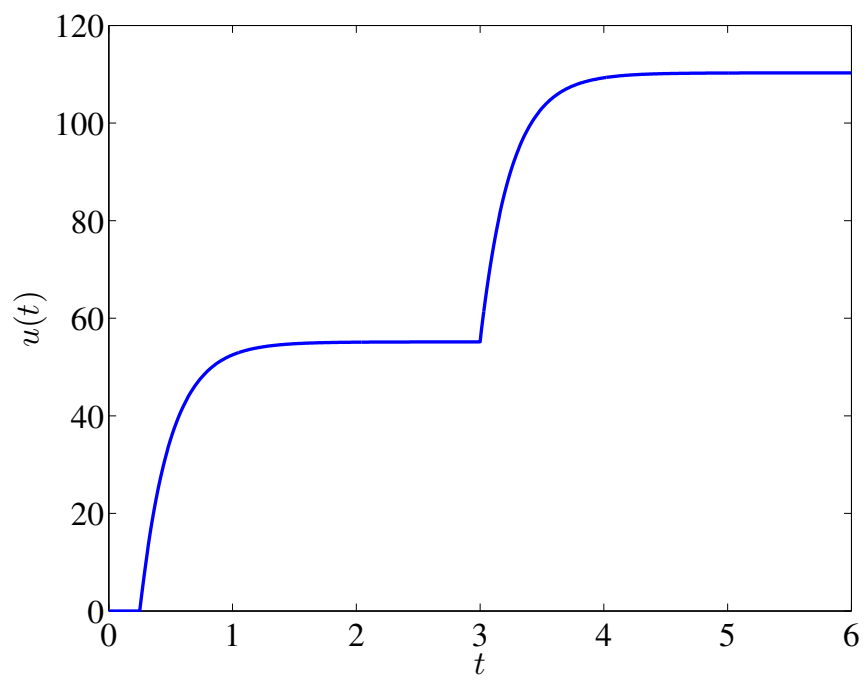

(b) Manipulated variable $u(t)$

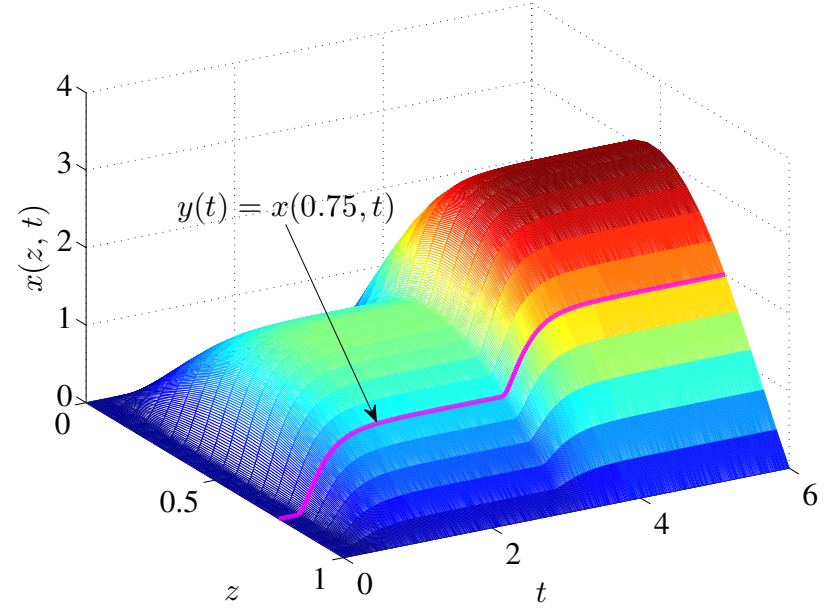

(c) State $x(z, t)$

Fig. 1: Tracking problem: Evolutions of the controlled output (Top), the manipulated variable (Middle) and the state (Bottom) in the case of a punctual output $y(t)=x(0.75, t)$.

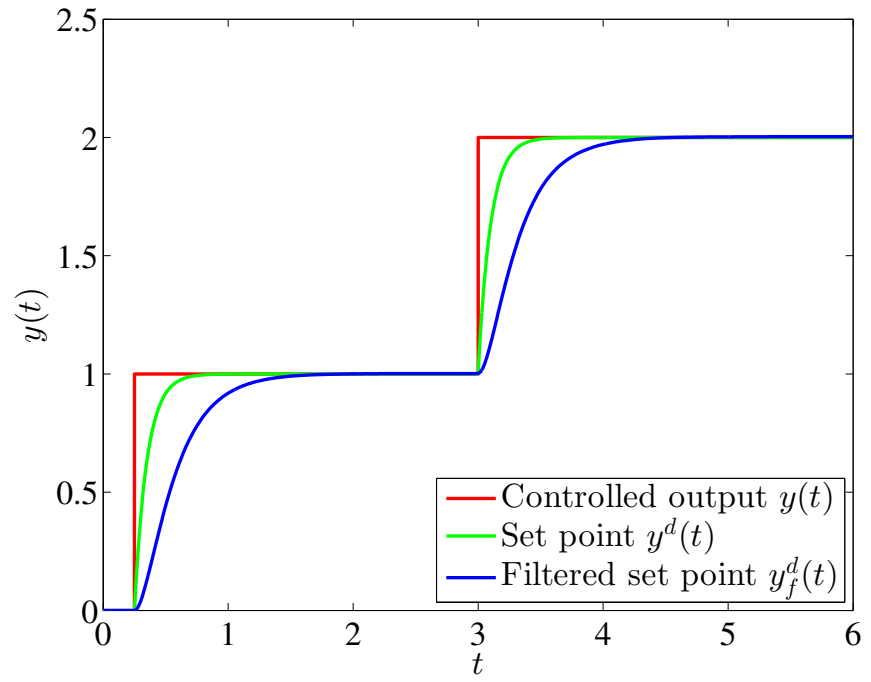

(a) Controlled output $y(t)$

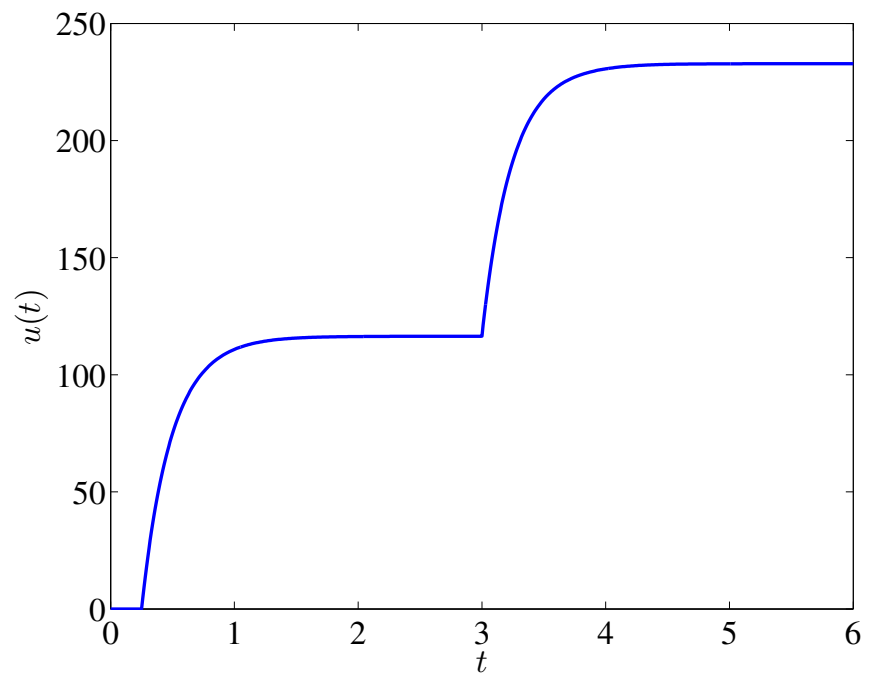

(b) Manipulated variable $u(t)$

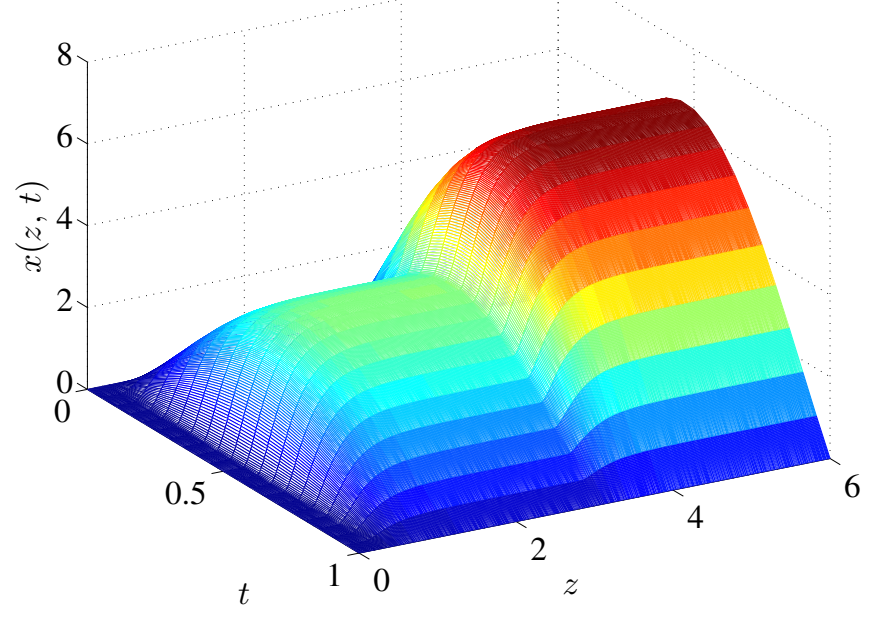

(c) State $x(z, t)$

Fig. 2: Tracking problem: Evolutions of the controlled output (Top), the manipulated variable (Middle) and the state (Bottom) in the case of a spatial weighted average output $y(t)=\int_{0}^{1} z x(z, t) d z$. 


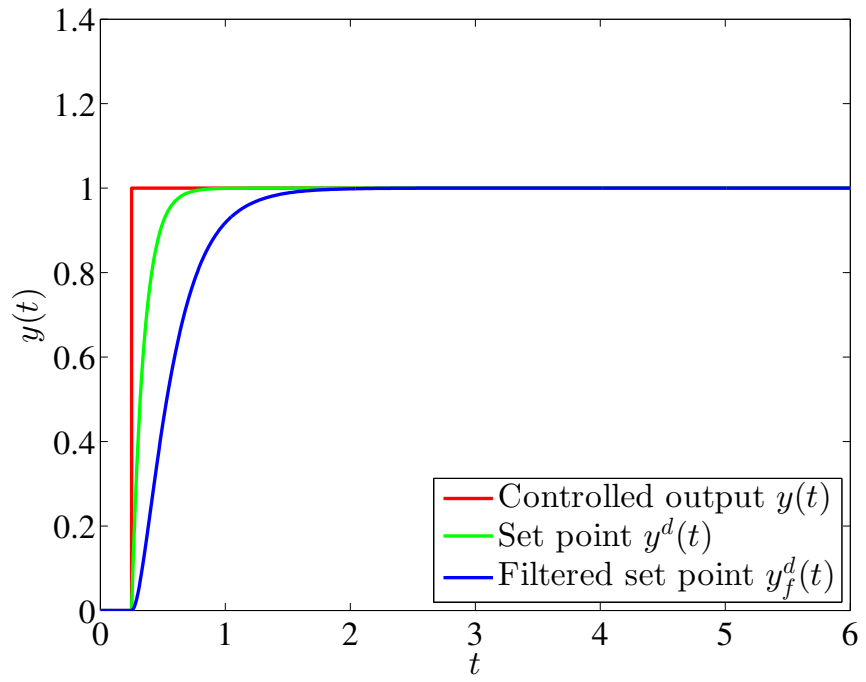

(a) Controlled output $y(t)$

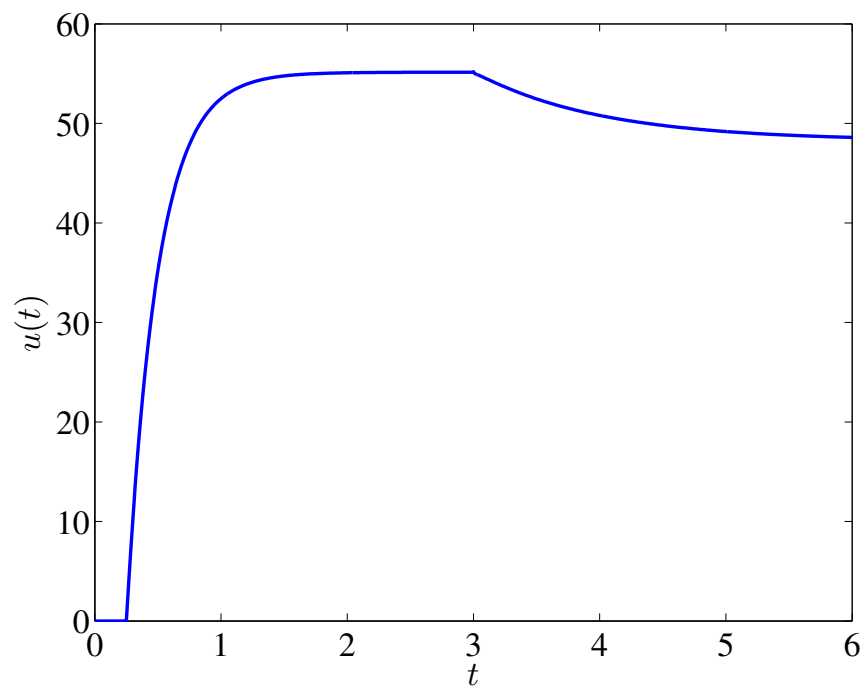

(b) Manipulated variable $u(t)$

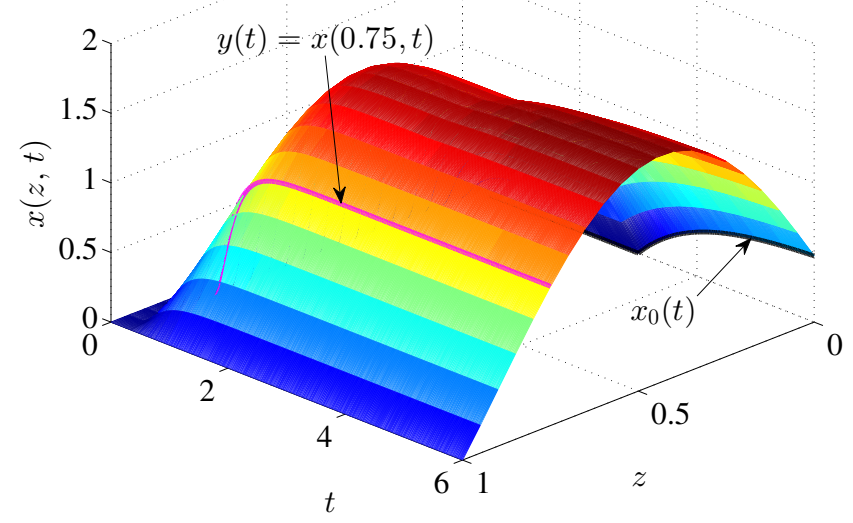

(c) State $x(z, t)$

Fig. 3: Disturbance rejection: Evolutions of the controlled output (Top), the manipulated variable (Middle) and the state (Bottom) in the case of a punctual output $y(t)=x(0.75, t)$.

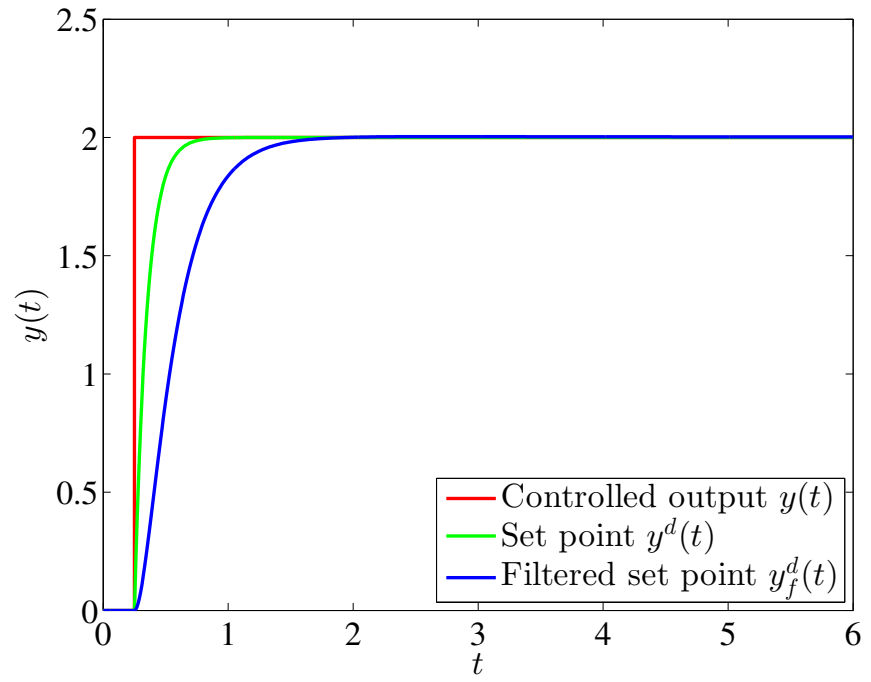

(a) Controlled output $y(t)$

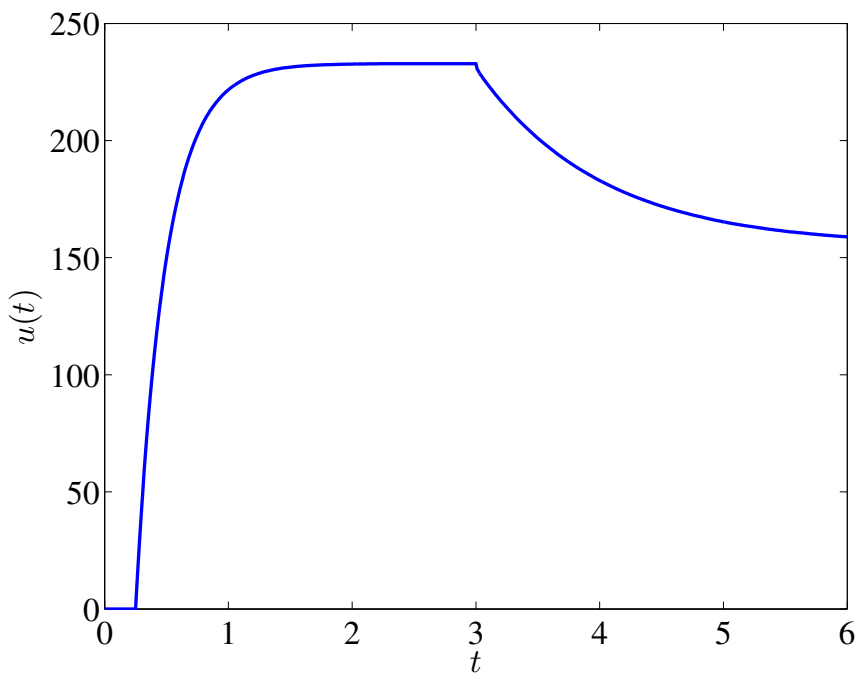

(b) Manipulated variable $u(t)$

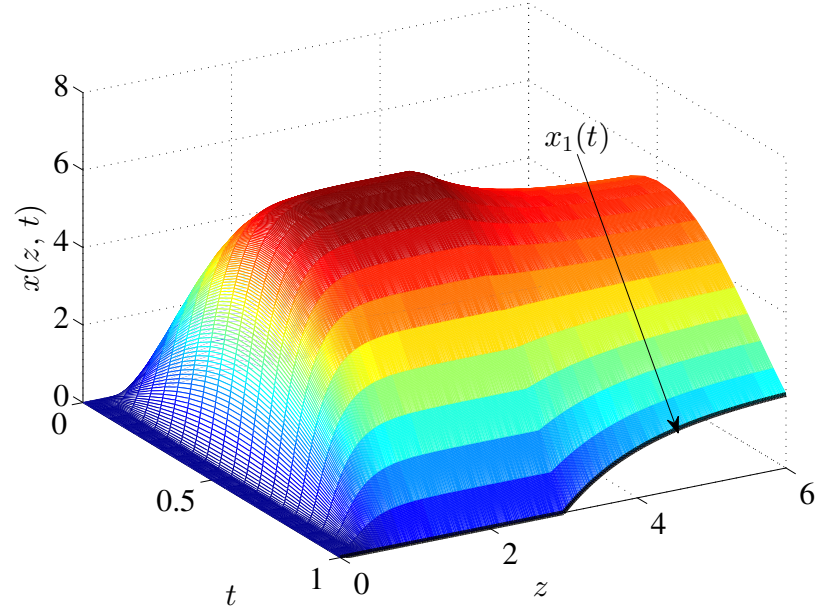

(c) State $x(z, t)$

Fig. 4: Disturbance rejection: Evolutions of the controlled output (Top), the manipulated variable (Middle) and the state (Bottom) in the case of a spatial weighted average output $y(t)=\int_{0}^{1} z x(z, t) d z$. 